\title{
Hiperparatiroidismo primario por adenoma gigante de paratiroides. Abordaje quirúrgico selectivo
}

Primary hyperparathyroidism caused by giant parathyroid adenoma. Selective surgical approach

Hiperparatireoidismo primário devido a um adenoma gigante da paratireoide. Abordagem cirúrgica seletiva

Daniel González González¹, Cecilia Chambón², Gustavo Armand Ugón³

\section{Resumen}

Introducción: el hiperparatiroidismo primario por un adenoma gigante de paratiroides es infrecuente. Los adenomas de mayor tamaño reportados ocurrieron sobre paratiroides ectópicas (mediastinales). Comparte con el carcinoma su gran tamaño y elevadas cifras de calcemia y de hormona paratiroidea, hecho que dificulta el diagnóstico. Su tratamiento quirúrgico es la paratiroidectomía mediante una cervicotomía transversa centrada en la región infrahioidea.

Objetivo: presentar un caso clínico de hiperparatiroidismo primario por un adenoma gigante de paratiroides tratado quirúrgicamente mediante un abordaje selectivo.

Caso clínico: paciente de 53 años, sexo femenino, con antecedentes de litiasis ureteral, dolores óseos y tumoración infrahiodea de $4 \mathrm{~cm}$ de diámetro que imagenológicamente presentó las características de un adenoma paratiroideo inferior izquierdo. La valoración funcional confirmó hiperparatiroidismo. Con diagnóstico de hiperparatiroidismo primario por adenoma gigante se trató quirúrgicamente a través de una incisión pequeña y centrada en la tumoración, realizándose la paratiroidectomía inferior izquierda con la cual remitió la sintomatología y normalizó la funcionalidad paratiroidea.

Discusión: el hiperparatiroidismo primario por adenoma gigante de paratiroides tiene indicación quirúrgica y es curativo. El caso presentado demuestra la factibilidad y seguridad de un abordaje selectivo a lo que suma una menor afectación cosmética, dejando indemne la logia tiroidea contralateral ante futuras cirugías sobre ésta.

Palabras clave: Hiperparatiroidismo primario

Neoplasias de las paratiroides

Adenoma paratiroideo gigante

Paratireoidectomía

Key words: $\quad$ Hyperparathyroidism, primary

Parathyroid neoplasms

Giant parathyroid adenoma

Parathyroidectomy

1. Prof. Titular de Clínica Quirúrgica 3.

2. Asistente de Clínica Quirúrgica 3.

3. Prof. Adjunto de Clínica Quirúrgica 3.

Clínica Quirúrgica 3. Facultad de Medicina. Universidad de la República. Hospital Maciel. Montevideo. Uruguay.

Correspondencia: Daniel González. Correo electrónico: danielalfredogg@gmail.com.

Recibido: 20/5/2020

Aprobado: 13/7/2020

Attribution-NonCommercial 4.0 International (CC BY-NC 4.0) 


\section{Introducción}

El adenoma gigante de paratiroides es una variedad poco frecuente dentro de los adenomas paratiroideos. Su diagnóstico se establece cuando el peso de la glándula patológica es superior a 3,5 g. En la serie de 300 adenomas paratiroides comunicada por Spanheimer ${ }^{(1)}$, los gigantes representaron el 5\%. Los mayores especímenes resecados y reportados en la literatura pesaron 220 y $145 \mathrm{~g}$, respectivamente, y ambos fueron mediastinales $^{(2,3)}$.

Se acompañan de manifestaciones clínicas de hiperparatiroidismo y habitualmente de valores de calcemia y hormona paratiroidea muy elevados. Su tratamiento es quirúrgico y su abordaje una cervicotomía transversa centrada en la región infrahiodea.

El objetivo del trabajo es la presentación de un caso clínico de hiperparatiroidismo primario por un adenoma gigante de paratiroides tratado quirúrgicamente mediante un abordaje selectivo.

\section{Caso clínico}

Paciente de 53 años, sexo femenino, con antecedentes de hipotiroidismo, litiasis ureteral izquierda y dolores óseos de cinco años de evolución.

Consultó por tumoración de región infrahioidea de dos años de evolución de crecimiento lento.

Paciente clínicamente en eutiroidismo destacándose del examen tumoración infrahiodea a predominio izquierdo de $4 \mathrm{~cm}$ de diámetro mayor.

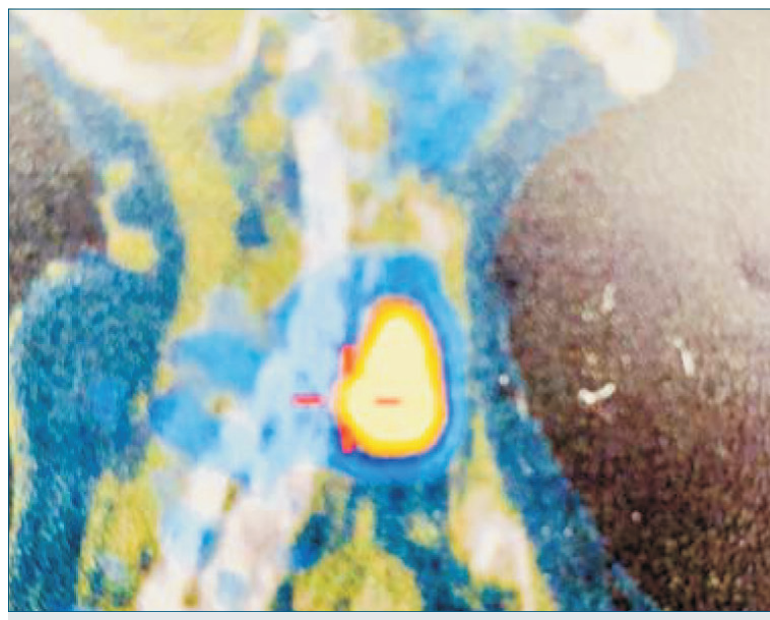

Figura 1. Centellograma Spect CT que evidencia la hipercaptación paratiroidea.

Se realizó ecografía de cuello que informó: tumoración sólida heterogénea de 53 por $21 \mathrm{~mm}$ de contornos lobulados que se relaciona con el polo inferior del lóbulo izquierdo de la tiroides con el que presenta un plano de clivaje y que se extiende hacia el mediastino. Desplaza el paquete yúgulo carotídeo y comprime el esófago.

Centellograma paratiroideo con Spect CT: área de hipercaptación anormal del MIBI localizada en la proyección del tercio inferior del lóbulo tiroides izquierdo con extensión posterior e inferior. En suma: imagen nodular hipermetabólica compatible con un adenoma paratiroideo inferior izquierdo (figura 1).

Calcemia de 12,86 mg/dl. PTHi de 472,8 ng/L.

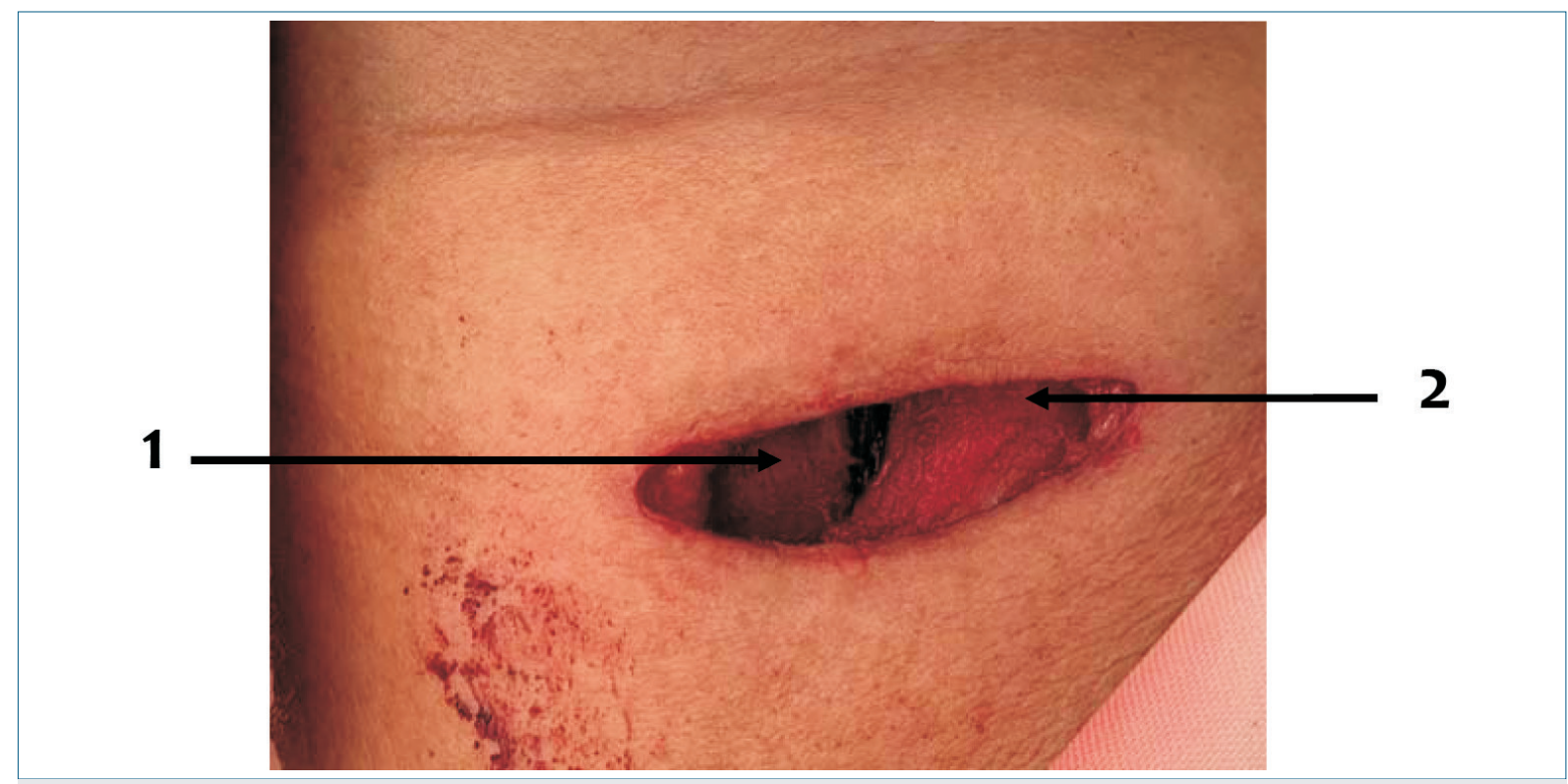

Figura 2. Cervicotomía izquierda. 1: músculos infrahiodeos. 2: músculo esternocleidomastoideo. 


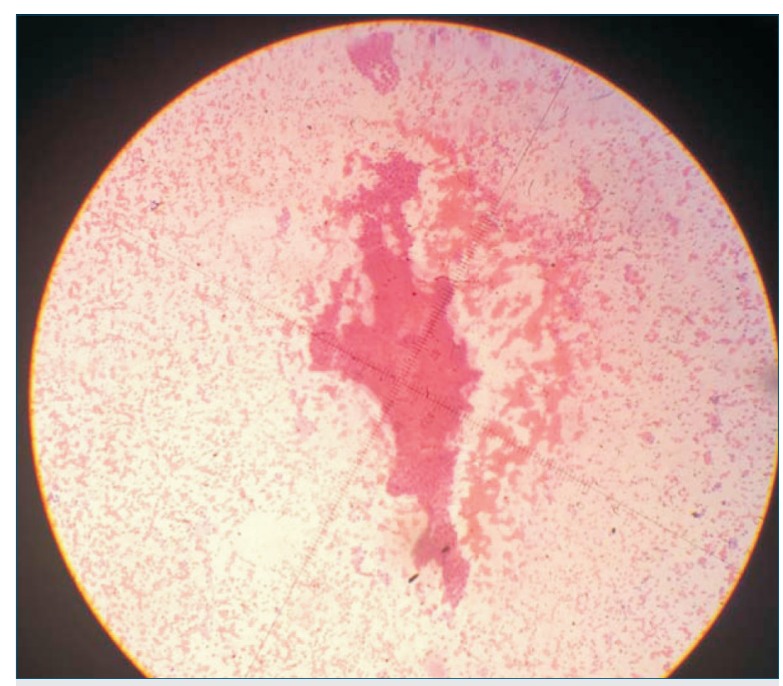

Figura 3. Estudio anatomopatológico extemporáneo con tinción de hematoxilina eosina que confirma presencia de células paratiroideas.

Con diagnóstico de hiperparatiroidimo primario se operó de coordinación mediante un abordaje selectivo centrado en la tumoración y una incisión de $4 \mathrm{~cm}$ de longitud (figura 2). Se realizó la paratiroidectomía inferior izquierda confirmada por estudio anatomopatológico extemporáneo (figura 3).

El estudio diferido de la pieza informó macroscopía: paratiroides de $16 \mathrm{~g}$ de peso y un tamaño de 50 por 30 por $15 \mathrm{~mm}$ (figura 4 ).

Microscopía: adenoma paratiroideo.

Buena evolución posoperatoria con desaparición de la sintomatología ósea, normalización de la calcemia y descenso a las 24 horas de PTHi a cifras de $148 \mathrm{ng} / \mathrm{L}$ y normalización al mes.

\section{Discusión}

Los adenomas paratiroideos gigantes son infrecuentes ${ }^{(4)}$, habiéndose demostrado en muchos de ellos mutaciones tipo MEN1 y HRPT2 ${ }^{(5)}$.

$\mathrm{Su}$ presentación clínica es variable. Algunos son diagnosticados por síntomas de hipercalcemia, otros por tumoración cervical o mediastinal (paratiroides ectópicas) y los más grandes agregan síntomas de compresión locorregional ${ }^{(6,7)}$. Es excepcional que no presenten síntomas de hipercalcemia dado que en general asocian valores elevados de calcemia y PTH -vinculadas a su tamaño-y en consecuencia con la gravedad de la sintomatología, así como el nivel de hipocalcemia en el posoperatorio luego de su resección completa ${ }^{(8,9)}$, que alcanzan cifras de $13 \%$ e incluso ser motivo de reingreso hospitalario $^{(1)}$. Spanheimer ${ }^{(1)}$ demostró la existencia de diferencias estadísticamente significativas en cuanto a niveles

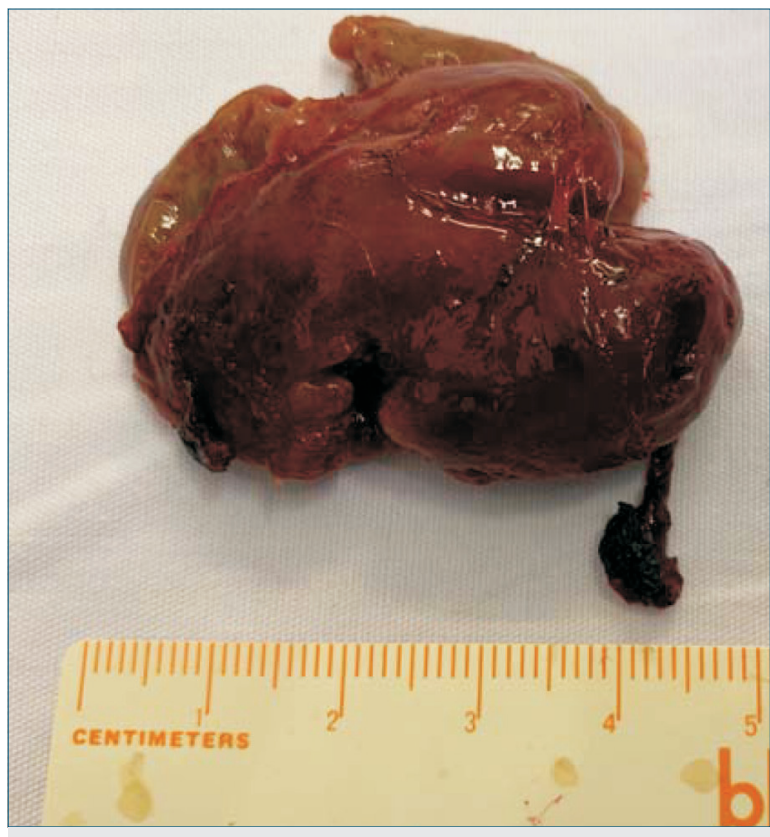

Figura 4. Pieza de resección quirúrgica.

de calcemia y PTH entre adenomas paratiroideos menores a 3,5 g y los gigantes, y además que el 93\% de los hiperparatiroidismos primarios por adenomas gigantes son determinados por una sola glándula patológica, como pensamos es el caso presentado, ya que a los 30 días normalizó las cifras de PTHi y calcemia.

El carcinoma de paratiroides comparte con los adenomas gigantes tres características -tamaño, valores elevados de calcemia y PTH- que determinan un verdadero desafío diagnóstico, especialmente si el carcinoma no presenta invasión a estructuras locorregionales o diseminación a distancia ${ }^{(10)}$.

Los estudios imagenológicos (ecografía y centellograma con tecnecio 99 sestamibi) permiten en el $87 \%$ de los casos localizar correctamente la o las glándulas patológicas ${ }^{(1)}$ y constituirse de esta manera en una herramienta esencial para la planificación de la cirugía, especialmente si se plantea un abordaje selectivo.

La incisión clásica y patrón oro para el tratamiento del hiperpartiroidismo primario eutópico es la cervicotomía arciforme de Kocher ${ }^{(11)}$. A través de ella se accede a la logia tiroidea, y luego de la movilización del lóbulo tiroideo correspondiente, se accede a la paratiroides patológica. Este abordaje puede evitarse de no existir una enfermedad tiroidea concomitante y con indicación quirúrgica, especialmente considerando futuros tratamientos quirúrgicos sobre la región, ya que es bien conocido el proceso adherencial que produce localmente, hecho que dificultaría una reintervención. El abordaje selectivo tiene la ventaja de ser seguro, requerir una menor incisión con el con- 
siguiente beneficio cosmético y dejar, como lo expresáramos anteriormente, indemne la logia tiroidea contralateral, lo que implica menores probabilidades de complicaciones en eventuales cirugías sobre la región ${ }^{(12-16)}$.

Por lo anterior, los abordajes con incisiones pequeñas y centradas en la topografía de la paratiroides a resecar ofrecen todas las ventajas para realizarlo. El desafío lo constituye la identificación preoperatoria de la glándula y su marcado. El caso comunicado no presentó dicha dificultad dado que su tamaño permitió su palpación sin dificultades, a diferencia de lo que ocurre con los adenomas no gigantes ${ }^{(13)}$. En otras circunstancias la ecografía realizada en el preoperatorio inmediato y en posición quirúrgica constituye una valiosa herramienta que presenta una sensibilidad y especificidad de $75 \%$ y $85 \%$ respectivamente $^{(17)}$.

La paratiroidectomía resuelve la enfermedad. Con ella remite la sintomatología y se normalizan la calcemia y PTHi, como ocurrió en el caso que comunicamos.

\section{Summary}

Introduction: primary hyperparathyroidism caused by giant parathyroid adenoma is a rather unusual condition. Reported large adenomas occurred in ectopic parathyroid glands (mediastinal). Just like carcinomas, they are large, present high calcemia and parathyroid hormone values, what complicates diagnosis. Surgical treatment consists in parathyroidectomy by means of transversal cervicotomy around the infrahyoid region.

Objective: the study presents the clinical case of primary hyperparathyroidism caused by giant parathyroid adenoma that was treated by selective surgery approach.

Clinical case: 53 year-old female patient with a history of uretheral lithiasis, bone pain and 4-cm-diameter infrahyoid tumor. Imagenology studies revealed the characteristics of lower left parathyroid adenoma. Functional assessment confirmed hyperparathyroidism.

Upon the diagnosis of primary hyperparathyroidism caused by giant parathyroid adenoma it was surgically addressed by means of a small cut around the tumour and performing a lower left parathyroidectomy, what resulted in the remission of symptoms and normalized parathyroid functionality.

Discussion: primary hyperparathyroidism caused by giant parathyroid adenoma has an indication for surgery and is therapeutic. The case presented shows the feasibility and safety of a selective approach, as well as its smaller cosmetic harm, managing to keep the contralateral thyroid loggia intact, in view of future surgeries involving it.

\section{Resumo}

Introdução: o hiperparatireoidismo primário devido a adenoma de paratireoide gigante é raro. Os maiores adenomas relatados ocorreram em paratireoides ectópicas (mediastinais). Compartilha com o carcinoma seu grande tamanho e altos níveis de cálcio e hormônio da paratireoide, o que torna o diagnóstico difícil. Seu tratamento cirúrgico é a paratireoidectomia por meio de cervicotomia transversa com foco na região infra-hióidea.

Objetivo: apresentar um caso clínico de hiperparatireoidismo primário por adenoma gigante da paratireoide tratado cirurgicamente por abordagem seletiva.

Caso clínico: paciente do sexo feminino, 53 anos, com história de litíase ureteral, dor óssea e tumor infra-hióideo de $4 \mathrm{~cm}$ de diâmetro que apresentava características de imagem de adenoma de paratireoide inferior esquerdo. A avaliação funcional confirmou hiperparatireoidismo.

Com diagnóstico de hiperparatireoidismo primário por adenoma gigante, foi tratada cirurgicamente por meio de pequena incisão focada no tumor, realizando paratireoidectomia inferior esquerda com remissão dos sintomas e normalização da funcionalidade da paratireoide.

Discussão: o hiperparatireoidismo primário devido ao adenoma gigante da paratireoide tem indicação cirúrgica e é curativo. O caso apresentado demonstra a viabilidade e segurança de uma abordagem seletiva que apresenta um menor envolvimento estético, deixando o espaço contralateral da tireoide sem danos para futuras cirurgias.

\section{Bibliografía}

1. Spanheimer PM, Stoltze AJ, Howe JR, Sugg SL, Lal G, Weigel RJ. Do giant parathyroid adenomas represent a distinct clinical entity? Surgery 2013; 154(4):714-8.

2. Garuna Murthee K, Tay WL, Soo KL, Swee DS. A migratory mishap: giant mediastinal parathyroid adenoma. Am J Med 2018; 131(5):512-6.

3. Cakmak H, Tokat AO, Karasu S, Özkan M. Giant mediastinal parathyroid adenoma. Tuberk Toraks 2011; 59(3):263-5.

4. Lalanne-Mistrih ML, Ognois-Ausse P, Goudet $\mathbf{P}$, Cougard P. Giant parathyroid tumors: characterization of 26 glands weighing more than 3.5 grams. Ann Chir 2002; 127(3):198-202.

5. Sulaiman L, Nilsson IL, Juhlin CC, Haglund F, Höög A, Larsson C, et al. Genetic characterization of large parathyroid adenomas. Endocr Relat Cancer 2012; 19(3):389-407.

6. Power C, Kavanagh D, Hill AD, O'Higgins N, McDermott E. Unusual presentation of a giant parathyroid adenoma: report of a case. Surg Today 2005; 35(3):235-7. 
7. Maciá C, Ronzón A, Botas P, Castaño G. Hipercalcemia y disfonía por adenoma gigante de paratiroides. Endocrinol Nutr 2005; 52(4):189-90.

8. Garas G, Poulasouchidou M, Dimoulas A, Hytiroglou P, Kita M, Zacharakis E. Radiological considerations and surgical planning in the treatment of giant parathyroid adenomas. Ann R Coll Surg Engl 2015; 97(4):e64-6.

9. Neagoe RM, Sala DT, Borda A, Mogoantã CA, Mûhlfay G. Clinicopathologic and therapeutic aspects of giant parathyroid adenomas - three case reports and short review of the literature. Rom J Morphol Embryol 2014; 55(2 Suppl):669-74.

10. Araujo Castro M, Abad López A, Martín Fragueiro L, Palacios García N. Giant parathyroid adenoma: differential aspects compared to parathyroid carcinoma. Endoc Diab Metab Case Rep 2017;pii17-0041.

11. Aygün N, Uludag M. Surgical treatment of primary hyperparathyroidism: which therapy to whom?. Sisli Eftal Hastan Tip Bul 2019; 53(3):201-4.

12. Al-Hassan MS, Mekhaimar M, El Ansari W, Darweesh A, Abdelaal A. Giant parathyroid adenoma: a case report and review of the literature. J Med Case Rep 2019; 13(1):332.
13. Mantzoros I, Kyriakidou D, Galanos-Demiris K, Chatzakis C, Parpoudi S, Sapidis N, et al. A rare case of primary hyperparathyroidism caused by a giant solitary parathyroid adenoma. Am J Case Rep 2018; 19:1334-7.

14. Haldar A, Thapar A, Khan S, Jenkins S. Day-case minimally invasive excision of a giant mediastinal parathyroid adenoma. Ann R Coll Surg Engl 2014; 96(5):e21-3.

15. Meyer-Rochow GY, Sidhu SB, Delbridge LW, Grodski S, Sywak MS. Can giant parathyroid adenomas be safely removed by minimally invasive parathyroidectomy. ANZ J Surg 2007; 77(Suppl 1):A23.

16. Christakis I, Michas S, Mouziouras V, Augoustis C, Dimas S. Minimally invasive techniques for giant parathyroid tumours; An oxymoron?. Hellenic J Surg 2012; 84(4):220-4.

17. Sahsamanis G, Gkouzis K, Samaras S, Pinialidis D, Dimitrakopoulos G. Surgical management of a giant parathyroid adenoma through minimal invasive parathyroidectomy. A case report. Int J Surg Case Rep 2017; 31:262-5.

\section{Contribución de autores}

Daniel González, https://orcid.org/ 0000-0003-3916-9201. Concepción, diseño, redacción y revisión crítica. Cecilia Chambón, https://orcid.org/ 0000-0001-9388-8886. Diseño y revisión crítica.

Gustavo Armand Ugón, https://orcid.org/ 0000-0003-2636-0867. Concepción, diseño y revisión crítica 